\title{
SOME HARDY-TYPE INEQUALITIES FOR THE GENERALIZED BAOUENDI-GRUSHIN OPERATORS*
}

\author{
PENGCHENG NIU, YANXIA CHEN** and YAZHOU HAN \\ Department of Applied Mathematics, Northwestern Polytechnical University, Xi'an, Shaanxi, \\ People's Republic of China, 710072
}

(Received 7 October, 2003; accepted 22 March, 2004)

\begin{abstract}
In this paper, we prove some Hardy-type inequalities for the degenerate operators, $L_{p, \alpha} u=\operatorname{div}_{L}\left(\left|\nabla_{L} u\right|^{p-2} \nabla_{L} u\right)$, where $\nabla_{L} u=\left(\frac{\partial u}{\partial z_{1}}, \ldots, \frac{\partial u}{\partial z_{n}},|z|^{\alpha} \frac{\partial u}{\partial t_{1}}, \ldots,|z|^{\alpha} \frac{\partial u}{\partial t_{m}}\right)$. These inequalities are established for the whole space, the pseudo-ball and the external domain of the pseudo-ball. We also give a generalization of a result in [8]. Finally, a sharp inequality for $L_{\alpha}=L_{2, \alpha}$ is obtained.
\end{abstract}

2000 Mathematics Subject Classification. 35H20, 47F05.

1. Introduction. The generalized Baouendi-Grushin operator is of the form

$$
L_{\alpha}=\Delta_{z}+|z|^{2 \alpha} \Delta_{t}
$$

where $\alpha>0, z=\left(z_{1}, z_{2}, \ldots, z_{n}\right) \in \mathbb{R}^{n}, t=\left(t_{1}, t_{2}, \ldots, t_{m}\right) \in \mathbb{R}^{m}, \Delta_{z}, \Delta_{t}$ are the Laplacians on $\mathbb{R}^{n}, \mathbb{R}^{m}$, respectively.

The related $p$-degenerate subelliptic operator is

$$
L_{p, \alpha}=\operatorname{div}_{L}\left(\left|\nabla_{L} u\right|^{p-2} \nabla_{L} u\right)
$$

where $\nabla_{L}=\left(\frac{\partial}{\partial z_{1}}, \ldots, \frac{\partial}{\partial z_{n}},|z|^{\alpha} \frac{\partial}{\partial t_{1}}, \ldots,|z|^{\alpha} \frac{\partial}{\partial t_{m}}\right)=\left(Z_{1}, \ldots Z_{n}, Z_{n+1}, \ldots, Z_{n+m}\right)$ is the generalized gradient and

$\operatorname{div}_{L}\left(u_{1}, \ldots, u_{n}, u_{n+1}, \ldots, u_{n+m}\right)=\frac{\partial u_{1}}{\partial z_{1}}+\cdots+\frac{\partial u_{n}}{\partial z_{n}}+|z|^{\alpha} \frac{\partial u_{n+1}}{\partial t_{1}}+\cdots+|z|^{\alpha} \frac{\partial u_{n+m}}{\partial t_{m}}$.

The operator $L_{\alpha}$ was first studied by Baouendi [1] and Grushin [6] when $\alpha$ is a positive integer. It is evident that if $\alpha$ is not an even positive integer then $L_{\alpha}$ is not a sum of squares of vector fields. This typical class of degenerate elliptic partial differential operators has been widely considered by many authors (see [4], [5] and references therein). In particular, Garofalo [5] established a Hardy-type inequality for $L_{\alpha}$ by using a representation formula of functions. Zhang and Niu [8] obtained a general inequality for $L_{p, \alpha}$ via a Picone identity for the vector fields $\left\{Z_{1}, \ldots, Z_{n}, Z_{n+1}, \ldots, Z_{n+m}\right\}$.

For $(z, t) \in \mathbb{R}^{n} \times \mathbb{R}^{m}$, define the distance between $(z, t)$ and the origin $(0,0)$ as

$$
d(z, t)=\left(|z|^{2(\alpha+1)}+(\alpha+1)^{2}|t|^{2}\right)^{\frac{1}{2(\alpha+1)}} .
$$

*This work supported by National Natural Science Foundation of China and Natural Science Foundation of Shaanxi Province, China.

** Present address: Campus School of Fengtac District, Beijing 100071. 
The homogeneous dimension with respect to the dilation

$$
\delta_{\epsilon}(z, t)=\left(\epsilon z, \epsilon^{\alpha+1} t\right), \epsilon>0
$$

is

$$
Q=n+m(\alpha+1)
$$

We will give in Section 2 some Hardy-type inequalities on some special domains in $\mathbb{R}^{n} \times \mathbb{R}^{m}$. The main approach we use is the appropriate choice of various auxiliary functions and the use of the Picone-type identity in [8].

The generalized Picone identities for elliptic operator systems in the Euclidean case and their applications were discussed in [7]. In Section 3 we generalize it to the degenerate elliptic operator system. It helps us to derive a Hardy-type inequality for $L_{p, \alpha}$ that is more general than the one in [8].

Section 4 is devoted to a sharp Hardy-type inequality for $L_{\alpha}$. The method of proof is similar to one used in [3].

2. Hardy-type inequalities on some special domains. The open pseudo-ball of radius $R$ and centred at the origin $(0,0) \in \mathbb{R}^{n} \times \mathbb{R}^{m}$ is denoted by

$$
B_{R}=\left\{(z, t) \in \mathbb{R}^{n} \times \mathbb{R}^{m} \mid d(z, t)<R\right\},
$$

and $\partial B_{R}$ is the boundary of $B_{R}$.

For the convenience of the reader, we begin by quoting two known results from $[8]$.

LEMma 2.1. For the differentiable functions $v>0$ and $u \geq 0$ on $\Omega \subset \mathbb{R}^{n+m}$, denote

$$
\begin{aligned}
& Q_{p}(u, v)=\left|\nabla_{L} u\right|^{p}+(p-1) \frac{u^{p}}{v^{p}}\left|\nabla_{L} v\right|^{p}-p \frac{u^{p-1}}{v^{p-1}}\left|\nabla_{L} v\right|^{p-2} \nabla_{L} u \cdot \nabla_{L} v, \\
& R_{p}(u, v)=\left|\nabla_{L} u\right|^{p}-\left|\nabla_{L} v\right|^{p-2} \nabla_{L}\left(\frac{u^{p}}{v^{p-1}}\right) \nabla_{L} v .
\end{aligned}
$$

Then

$$
Q_{p}(u, v)=R_{p}(u, v) \geq 0 .
$$

LEMMA 2.2. For some $\lambda>0$ and a nonnegative weight function $g$ on $\Omega$, if $0<v \in C_{0}^{\infty}$ satisfying

$$
-L_{p, \alpha} v \geq \lambda g v^{p-1} \quad \text { on } \Omega
$$

then for $u \geq 0$,

$$
\int_{\Omega}\left|\nabla_{L} u\right|^{p} \geq \lambda \int_{\Omega} g|u|^{p} .
$$

These are Theorems 3 and 4 in [8], respectively.

The first result is the Hardy inequality on the $B_{R} \backslash\{(0,0)\}$. 
THEOREM 2.1. Let $\delta(z, t)=d\left((z, t), \partial B_{R}\right)$, for any $(z, t) \in B_{R}$, and $\Omega=B_{R} \backslash\{(0,0)\}$. Then, for $p>1$, we have

$$
\int_{\Omega}\left|\nabla_{L} u\right|^{p} \geq\left(\frac{p-1}{p}\right)^{p} \int_{\Omega} \frac{|z|^{p \alpha}}{d^{p \alpha}} \frac{|u|^{p}}{\delta^{p}},
$$

for every $u \in C_{0}^{\infty}(\Omega)$, where $d=d(z, t)$.

Proof. Let $u \geq 0$. It is clear to

$$
\begin{aligned}
Z_{j} d & =Z_{j}\left(|z|^{2(\alpha+1)}+(\alpha+1)^{2}|t|^{2}\right)^{\frac{1}{2(\alpha+1)}}=d^{-2 \alpha-1}|z|^{2 \alpha} z_{j}, \quad j=1, \ldots, n, \\
Z_{j} d & =Z_{j}\left(|z|^{2(\alpha+1)}+(\alpha+1)^{2}|t|^{2}\right)^{\frac{1}{2(\alpha+1)}} \\
& =d^{-2 \alpha-1}|z|^{\alpha}(\alpha+1) t_{j}, \quad j=n+1, \ldots, n+m,
\end{aligned}
$$

therefore

$$
\begin{aligned}
\nabla_{L} d= & \left(d^{-2 \alpha-1}|z|^{2 \alpha} z_{1}, \ldots, d^{-2 \alpha-1}|z|^{2 \alpha} z_{n}\right. \\
& \left.\times d^{-2 \alpha-1}|z|^{\alpha}(\alpha+1) t_{1}, \ldots, d^{-2 \alpha-1}|z|^{\alpha}(\alpha+1) t_{m}\right), \\
\left|\nabla_{L} d\right|^{2}= & \left(d^{-2 \alpha-1}\right)^{2}\left[\sum_{j=1}^{n}\left(|z|^{2 \alpha} z_{j}\right)^{2}+\sum_{j=n+1}^{n+m}\left(|z|^{\alpha}(\alpha+1) t_{j}\right)^{2}\right] \\
= & \left(d^{-2 \alpha-1}\right)^{2}\left(|z|^{4 \alpha}|z|^{2}+|z|^{2 \alpha}(\alpha+1)^{2}|t|^{2}\right) \\
= & d^{-4 \alpha-2} d^{2(\alpha+1)}|z|^{2 \alpha} \\
= & \left(\frac{|z|^{\alpha}}{d^{\alpha}}\right)^{2}
\end{aligned}
$$

and

$$
\left|\nabla_{L} d\right|=\frac{|z|^{\alpha}}{d^{\alpha}}
$$

Using the notation

$$
\psi_{p, \alpha}=\frac{|z|^{p \alpha}}{d^{p \alpha}}
$$

it follows that

$$
\begin{aligned}
L_{p, \alpha} d & =\operatorname{div}_{L}\left(\left|\nabla_{L} d\right|^{p-2} \nabla_{L} d\right) \\
& =\psi_{p \alpha} \frac{d^{(\alpha+1)(p-2)}}{d^{(\alpha+1)(p-2)+1}}\left[Q+(\alpha+1)(p-2)+p \alpha \cdot 0-[(\alpha+1)(p-2)+1] \frac{d^{2(\alpha+1)}}{d^{2(\alpha+1)}}\right] \\
& \left.=\frac{|z|^{p \alpha}}{d^{p \alpha}} \frac{1}{d}[Q+(\alpha+1)(p-2)-(\alpha+1)(p-2)-1)\right] \\
& =\frac{(Q-1)|z|^{p \alpha}}{d^{p \alpha+1}} .
\end{aligned}
$$


Choosing $v=\delta^{\beta}=(R-d)^{\beta}=f(d), \beta=\frac{p-1}{p}$, we get

$$
\begin{aligned}
L_{p, \alpha}(f(d)) & =\operatorname{div}_{L}\left(\left|\nabla_{L} f(d)\right|^{p-2} \nabla_{L} f(d)\right) \\
& =\operatorname{div}_{L}\left(\left|f^{\prime}(d)\right|^{p-2} f^{\prime}(d)\left|\nabla_{L} d\right|^{p-2} \nabla_{L} d\right) \\
& =\left|f^{\prime}(d)\right|^{p-2} f^{\prime}(d) \operatorname{div}_{L}\left(\left|\nabla_{L} d\right|^{p-2} \nabla_{L} d\right)+\nabla_{L}\left(\left|f^{\prime}(d)\right|^{p-2} f^{\prime}(d)\right) \cdot\left|\nabla_{L} d\right|^{p-2} \nabla_{L} d \\
& =\left|f^{\prime}(d)\right|^{p-2}\left[f^{\prime}(d) L_{p, \alpha} d+(p-1) f^{\prime \prime}(d)\left|\nabla_{L} d\right|^{p}\right],
\end{aligned}
$$

and then

$$
\begin{aligned}
-L_{p, \alpha} v & =-\left|-\frac{p-1}{p} \delta^{-\frac{1}{p}}\right|^{p-2}\left[-\frac{p-1}{p} \delta^{-\frac{1}{p}} \frac{(Q-1)|z|^{p \alpha}}{d^{p \alpha+1}}+(p-1)\left(-\frac{p-1}{p^{2}}\right) \delta^{-\frac{p+1}{p}} \frac{|z|^{p \alpha}}{d^{p \alpha}}\right] \\
& =-\left(\frac{p-1}{p}\right)^{p-2} \delta^{-\frac{p-2}{p}} \frac{1}{d^{p \alpha}}\left[-\frac{(p-1)(Q-1)}{p}|z|^{p \alpha} \delta^{-\frac{1}{p}} \frac{1}{d}-\frac{(p-1)^{2}}{p^{2}}|z|^{p \alpha} \delta^{-\frac{p+1}{p}}\right] \\
& =\left(\frac{p-1}{p}\right)^{p-1} \delta^{-\frac{p-2}{p}} \delta^{-\frac{p+1}{p}} \frac{|z|^{p \alpha}}{d^{p \alpha}}\left[\frac{p-1}{p}+(Q-1) \frac{\delta}{d}\right] \\
& =\left(\frac{p-1}{p}\right)^{p-1}\left\{\frac{p-1}{p}+(Q-1) \frac{\delta}{d}\right\} \frac{|z|^{p \alpha}}{d^{p \alpha}} \frac{v^{p-1}}{\delta^{p}} \\
& \geq\left(\frac{p-1}{p}\right)^{p} \frac{|z|^{p \alpha}}{d^{p \alpha}} \frac{v^{p-1}}{\delta^{p}} .
\end{aligned}
$$

From Theorems 2 and 3 in [8], we obtain

$$
\int_{\Omega}\left|\nabla_{L} u\right|^{p} \geq\left(\frac{p-1}{p}\right)^{p} \int_{\Omega} \frac{|z|^{p \alpha}}{d^{p \alpha}} \frac{|u|^{p}}{\delta^{p}} .
$$

For general $u$, letting $u=u^{+}-u^{-}$yields the desired statement.

Now we consider the domain $\Omega=\mathbb{R}^{n+m} \backslash B_{R}$.

THEOREM 2.2. Let $\Omega=\mathbb{R}^{n+m} \backslash B_{R}$ and $1<p<Q$. Then, for every $u \in C_{0}^{\infty}(\Omega)$

$$
\int_{\Omega}\left|\nabla_{L} u\right|^{p} \geq C \int_{\Omega} \frac{|z|^{p \alpha}}{d^{p(\alpha+1)}} \frac{|u|^{p}}{d^{p}}=C \int_{\Omega} \frac{|z|^{p \alpha}}{d^{p \alpha}} \frac{|u|^{p}}{d^{2 p}},
$$

where $d=d(z, t)$.

Proof. It is enough to prove the claim for $u \geq 0$. Choosing

$$
v=\ln \left(\frac{d}{R}\right)^{\alpha}=f(d), \quad \text { where } R<d<+\infty, \alpha=p-Q,
$$

it follows that

$$
f^{\prime}(d)=\frac{\alpha}{d}=\frac{p-Q}{d}
$$

and

$$
f^{\prime \prime}(d)=-\frac{\alpha}{d^{2}}=\frac{Q-p}{d^{2}} .
$$


Thus,

$$
\begin{aligned}
-L_{p, \alpha} & =-\left|f^{\prime}(d)\right|^{p-2}\left[f^{\prime}(d) L_{p, \alpha} d+(p-1) f^{\prime \prime}(d)\left|\nabla_{L} d\right|^{p}\right] \\
& =-\frac{(Q-p)^{p-2}}{d^{p-2}}\left[\frac{(Q-1)(p-Q)|z|^{p \alpha}}{d^{p \alpha+2}}+(p-1)(Q-p) \frac{|z|^{p \alpha}}{d^{p \alpha+2}}\right] \\
& =-\frac{(Q-p)^{p-2}}{d^{p(\alpha+1)}}|z|^{p \alpha}\left[-(Q-p)^{2}\right] \\
& =(Q-p)^{p} \frac{|z|^{p \alpha}}{d^{p(\alpha+1)}} \\
& =(Q-p)^{p} \frac{|z|^{p \alpha}}{d^{p(\alpha+1)}} \frac{v^{p-1}}{v^{p-1}} .
\end{aligned}
$$

Since

$$
\lim _{d \rightarrow+\infty} \frac{v^{p-1}}{d^{p}}=\lim _{d \rightarrow+\infty}\left(\ln \left(\frac{d}{R}\right)^{\alpha}\right)^{p-1} d^{-p}=0,
$$

there exists a positive integer $N \geq R$, such that if $d>N$, then $\frac{v^{p-1}}{d^{p}}<1$. On the other hand, $\frac{v^{p-1}}{d^{p}}$ is continuous on the interval $[R, N]$, so we can find a positive constant $C^{\prime}$ such that $\frac{v^{p-1}}{d^{p}} \leq C^{\prime}$. Taking $C^{\prime \prime}=\max \left\{C^{\prime}, 1\right\}$, it shows that

$$
\frac{v^{p-1}}{d^{p}} \leq C^{\prime \prime}, \quad \text { i.e., } v^{p-1} \leq C^{\prime \prime} d^{p}
$$

if $d \geq R$.

Equation (2.7) becomes

$$
-L_{p, \alpha} v>(Q-p)^{p} \frac{|z|^{p \alpha}}{d^{p(\alpha+1)}} \frac{v^{p-1}}{C^{\prime \prime} d^{p}}=C \frac{|z|^{p \alpha}}{d^{p(\alpha+1)}} \frac{v^{p-1}}{d^{p}},
$$

where $C=\frac{(Q-p)^{p}}{C^{\prime \prime}}$. Using Theorem 3 in $[\mathbf{8}]$, we have

$$
\int_{\Omega}\left|\nabla_{L} u\right|^{p} \geq C \int_{\Omega} \frac{|z|^{p \alpha}}{d^{p(\alpha+1)}} \frac{|u|^{p}}{d^{p}}=C \int_{\Omega} \frac{|z|^{p \alpha}}{d^{p \alpha}} \frac{|u|^{p}}{d^{2 p}} .
$$

THEOREM 2.3. Let $\Omega=\mathbb{R}^{n+m} \backslash B_{R}$ and $p \geq Q$. Then the inequality

$$
\int_{\Omega}\left|\nabla_{L} u\right|^{p} \geq C \int_{\Omega} \frac{|z|^{p \alpha}}{d^{p \alpha}} \frac{|u|^{p}}{d^{p}\left(\ln \frac{d}{R}\right)^{p}}, \quad u \in C_{0}^{\infty}(\Omega),
$$

is valid.

Proof. As above, we only consider $u \geq 0$. The choice

$$
v=\left(\ln \frac{d}{R}\right)^{\alpha}=f(d), \quad \text { where } R<d<+\infty, 0<\alpha<1,
$$

yields

$$
f^{\prime}(d)=\frac{\alpha}{d}\left(\ln \frac{d}{R}\right)^{\alpha-1}
$$




$$
f^{\prime \prime}(d)=-\frac{\alpha}{d^{2}}\left(\ln \frac{d}{R}\right)^{\alpha-1}+\frac{\alpha(\alpha-1)}{d^{2}}\left(\ln \frac{d}{R}\right)^{\alpha-2},
$$

and then

$$
\begin{aligned}
-L_{p, \alpha} v= & -\left|f^{\prime}(d)\right|^{p-2}\left[(p-1) f^{\prime \prime}(d)\left|\nabla_{L} d\right|^{p}+f^{\prime}(d) L_{p, \alpha} d\right] \\
= & -\left|\frac{\alpha}{d}\left(\ln \frac{d}{R}\right)^{\alpha-1}\right|^{p-2}\left\{( p - 1 ) \frac { | z | ^ { p \alpha } } { d ^ { p \alpha } } \left(-\frac{\alpha}{d^{2}}\left(\ln \frac{d}{R}\right)^{\alpha-1}\right.\right. \\
& \left.\left.+\frac{\alpha(\alpha-1)}{d^{2}}\left(\ln \frac{d}{R}\right)^{\alpha-2}\right)+\frac{(Q-1)|z|^{p \alpha}}{d^{p \alpha+1}} \frac{\alpha}{d}\left(\ln \frac{d}{R}\right)^{\alpha-1}\right\} \\
= & -\frac{\alpha^{p-2}}{d^{p-2}}\left(\ln \frac{d}{R}\right)^{(\alpha-1)(p-2)}\left\{( p - 1 ) \frac { | z | ^ { p \alpha } } { d ^ { p \alpha + 2 } } \left(-\alpha\left(\ln \frac{d}{R}\right)^{\alpha-1}\right.\right. \\
& \left.\left.+\alpha(\alpha-1)\left(\ln \frac{d}{R}\right)^{\alpha-2}\right)+\frac{(Q-1)|z|^{p \alpha} \alpha}{d^{p \alpha+2}}\left(\ln \frac{d}{R}\right)^{\alpha-1}\right\} \\
= & -\frac{|z|^{p \alpha} \alpha^{p-1}}{d^{p(\alpha+1)}}\left(\ln \frac{d}{R}\right)^{\alpha(p-1)-p}\left[(\alpha-1)(p-1)+(Q-p) \ln \frac{d}{R}\right] .
\end{aligned}
$$

Noting $-\alpha^{p-1}(Q-p) \ln \frac{d}{R} \geq 0$, we get from (2.10) that

$$
-L_{p, \alpha} v \geq-\alpha^{p-1}(\alpha-1)(p-1) \frac{v^{p-1}}{\left(\ln \frac{d}{R}\right)^{p}} \frac{|z|^{p \alpha}}{d^{p(\alpha+1)}}=C \frac{v^{p-1}}{\left(\ln \frac{d}{R}\right)^{p}} \frac{|z|^{p \alpha}}{d^{p(\alpha+1)}},
$$

where $C=-\alpha^{p-1}(\alpha-1)(p-1)$. Using Theorem 3 in [8] gives

$$
\int_{\Omega}\left|\nabla_{L} u\right|^{p} \geq C \int_{\Omega} \frac{|z|^{p \alpha}}{d^{p(\alpha+1)}} \frac{|u|^{p}}{\left(\ln \frac{d}{R}\right)^{p}}=C \int_{\Omega} \frac{|z|^{p \alpha}}{d^{p \alpha}} \frac{|u|^{p}}{d^{p}\left(\ln \frac{d}{R}\right)^{p}} .
$$

This ends the proof.

We do not know how to prove Hardy-type inequalities for $L_{\alpha}$ or $L_{p, \alpha}$ on bounded or unbounded domains in $\mathbb{R}^{n} \times \mathbb{R}^{m}$ other than those discused above. This is an open question.

3. Generalized Picone-type identities and Hardy-type inequalities. Consider the following system of degenerate subelliptic operators

$$
\begin{aligned}
& p_{\gamma}[u]=\nabla_{L} \cdot\left(a(x) \Phi\left(\nabla_{L} u\right)\right)+c(x) \phi(u) \\
& P_{\gamma}[v]=\nabla_{L} \cdot\left(A(x) \Phi\left(\nabla_{L} v\right)\right)+C(x) \phi(v),
\end{aligned}
$$

where $\phi(s)=|s|^{\gamma-1} s, s \in \mathbb{R}, \Phi(\xi)=|\xi|^{\gamma-1} \xi, \xi \in \mathbb{R}^{n} \times \mathbb{R}^{m}, \gamma>0, \nabla_{L}$ is the generalized gradient. The boundary $\partial \Omega$ of the domain $\Omega \subset \mathbb{R}^{n} \times \mathbb{R}^{m}$ is piecewise smooth. The functions $a(x), c(x), A(x)$ and $C(x)$ in (3.1) and (3.2) satisfy respectively: $a \in C^{1}\left(\bar{\Omega} ; \mathbb{R}_{+},\right), \quad A \in C^{1}\left(\bar{\Omega} ; \mathbb{R}_{+}\right), c \in C(\bar{\Omega} ; \mathbb{R}), C \in C(\bar{\Omega} ; \mathbb{R})$. 
Define

$$
\begin{gathered}
D_{p_{\gamma}(\Omega)}=\left\{u \in C^{1}(\bar{\Omega} ; \mathbb{R}): a(x) \Phi\left(\nabla_{L} u\right) \in C^{1}(\Omega ; \mathbb{R}) \bigcap C(\bar{\Omega} ; \mathbb{R})\right\} ; \\
D_{P_{\gamma}(\Omega)}=\left\{v \in C^{1}(\bar{\Omega} ; \mathbb{R}): A(x) \Phi\left(\nabla_{L} v\right) \in C^{1}(\Omega ; \mathbb{R}) \bigcap C(\bar{\Omega} ; \mathbb{R})\right\},
\end{gathered}
$$

respectively.

The following is a generalized Picone-type identity.

Lemma 3.1. Suppose that $u \in D_{p_{\gamma}(\Omega)}, v \in D_{P_{\gamma}(\Omega)}$ and $v \neq 0$ on $\Omega$. Then

$$
\begin{aligned}
\nabla_{L} \cdot\{ & \left.\frac{u}{\phi(v)}\left[\phi(v) a(x) \Phi\left(\nabla_{L} u\right)-\phi(u) A(x) \Phi\left(\nabla_{L} v\right)\right]\right\} \\
= & (a(x)-A(x))\left|\nabla_{L} u\right|^{\gamma+1}+(C(x)-c(x))|u|^{\gamma+1} \\
& +A(x)\left[\left|\nabla_{L} u\right|^{\gamma+1}+\gamma\left|\frac{u}{v} \nabla_{L} v\right|^{\gamma+1}-(\gamma+1)\left|\frac{u}{v} \nabla_{L} v\right|^{\gamma-1} \nabla_{L} u \cdot\left(\frac{u}{v} \nabla_{L} v\right)\right] \\
& +\frac{u}{\phi(v)}\left\{\phi(v) p_{\gamma}[u]-\phi(u) P_{\gamma}[v]\right\} .
\end{aligned}
$$

Proof. A direct calculation shows that

$\left(P_{1}\right)\left\{\begin{array}{l}\phi(s) \in C^{1}(\mathbb{R} \backslash\{0\} ; \mathbb{R}) \\ s \phi^{\prime}(s) \in C(\mathbb{R} ; \mathbb{R}) \\ s \phi^{\prime}(s)=\gamma \phi(s), s \in \mathbb{R} \\ \phi(s) \phi(t)=\phi(s t), s \in \mathbb{R} \text { and } t \in \mathbb{R} \\ \phi(-s)=-\phi(s), s \in \mathbb{R} \\ \phi(s)>0, s>0,\end{array}\right.$

$\left(P_{2}\right)\left\{\begin{array}{l}\Phi(\xi) \in C^{1}\left(\mathbb{R}^{n+m} \backslash\{0\} ; \mathbb{R}^{n+m}\right) \cap C\left(\mathbb{R}^{n+m} ; \mathbb{R}^{n+m}\right) \\ \Phi(-\xi)=-\Phi(\xi), \xi \in \mathbb{R}^{n+m} \\ \phi(s) \Phi(\xi)=\Phi(s \xi), s \in \mathbb{R} \text { and } \xi \in \mathbb{R}^{n+m} .\end{array}\right.$

Evidently, we have

$$
\begin{gathered}
u p_{\gamma}[u]=u \nabla_{L} \cdot\left(a(x) \Phi\left(\nabla_{L} u\right)\right)+c(x) u \phi(u) \\
=\nabla_{L}\left(u a(x) \Phi\left(\nabla_{L} u\right)\right)-\nabla_{L} u \cdot\left(a(x) \Phi\left(\nabla_{L} u\right)\right)+c(x) u \phi(u), \\
\nabla_{L}\left(\frac{u}{\phi(v)} \phi(v) a(x) \Phi\left(\nabla_{L} u\right)\right)=a(x) \nabla_{L} u \cdot \Phi\left(\nabla_{L} u\right)-c(x) u \phi(u)+\frac{u}{\phi(v)}\left(\phi(v) p_{\gamma}[u]\right),
\end{gathered}
$$

and

$$
\begin{aligned}
\nabla_{L} \cdot\left(u \phi(u) \frac{A(x) \Phi\left(\nabla_{L} v\right)}{\phi(v)}\right) \\
=\nabla_{L} u \cdot\left(\phi(u) \frac{A(x) \Phi\left(\nabla_{L} v\right)}{\phi(v)}\right)+u \phi^{\prime}(u) \nabla_{L} u \cdot \frac{A(x) \Phi\left(\nabla_{L} v\right)}{\phi(v)} \\
\quad+u \phi(u)\left(-\frac{\phi^{\prime}(v)}{\phi^{2}(v)} \nabla_{L} v\right) \cdot\left(A(x) \Phi\left(\nabla_{L} v\right)\right)+u \frac{\phi(u)}{\phi(v)} \nabla_{L} \cdot\left(A(x) \Phi\left(\nabla_{L} v\right)\right)
\end{aligned}
$$




$$
\begin{aligned}
= & A(x) \phi\left(\frac{u}{v}\right) \nabla_{L} u \cdot \Phi\left(\nabla_{L} v\right)+\gamma A(x) \phi\left(\frac{u}{v}\right) \nabla_{L} u \cdot \Phi\left(\nabla_{L} v\right) \\
& -\gamma \frac{u}{v} \phi\left(\frac{u}{v}\right) A(x) \nabla_{L} v \cdot \Phi\left(\nabla_{L} v\right)+\frac{u}{\phi(v)}\left(\phi(u) P_{\gamma}[v]\right)-C(x) u \phi(u) \\
= & A(x) \nabla_{L} u \cdot \Phi\left(\nabla_{L} u\right)-C(x) u \phi(u)-A(x)\left\{\nabla_{L} u \cdot \Phi\left(\nabla_{L} u\right)\right. \\
& \left.+\gamma\left(\frac{u}{v} \nabla_{L} v\right) \cdot \Phi\left(\frac{u}{v} \nabla_{L} v\right)-(\gamma+1) \nabla_{L} u \cdot \Phi\left(\frac{u}{v} \nabla_{L} v\right)\right\} \\
& +\frac{u}{\phi(v)}\left(\phi(u) P_{\gamma}[v]\right) .
\end{aligned}
$$

We immediately obtain

$$
\begin{aligned}
\nabla_{L} \cdot\{ & \left.\frac{u}{\phi(v)}\left[\phi(v) a(x) \Phi\left(\nabla_{L} u\right)-\phi(u) A(x) \Phi\left(\nabla_{L} v\right)\right]\right\} \\
= & (a(x)-A(x)) \nabla_{L} u \cdot \Phi\left(\nabla_{L} u\right)+(C(x)-c(x)) u \phi(u) \\
& +A(x)\left\{\nabla_{L} u \cdot \Phi\left(\nabla_{L} u\right)+\gamma\left(\frac{u}{v} \nabla_{L} v\right) \cdot \Phi\left(\frac{u}{v} \nabla_{L} v\right)\right. \\
& \left.-(\gamma+1) \nabla_{L} u \cdot \Phi\left(\frac{u}{v} \nabla_{L} v\right)\right\}+\frac{u}{\phi(v)}\left(\phi(v) p_{\gamma}[u]-\phi(u) P_{\gamma}[v]\right) \\
= & (a(x)-A(x))\left|\nabla_{L} u\right|^{\gamma+1}+(C(x)-c(x))|u|^{\gamma+1} \\
& +A(x)\left\{\left|\nabla_{L} u\right|^{\gamma+1}+\gamma\left|\frac{u}{v} \nabla_{L} v\right|^{\gamma+1}-(\gamma+1)\left|\frac{u}{v} \nabla_{L} v\right|^{\gamma-1} \nabla_{L} u \cdot\left(\frac{u}{v} \nabla_{L} v\right)\right\} \\
& +\frac{u}{\phi(v)}\left(\phi(v) p_{\gamma}[u]-\phi(u) P_{\gamma}[v]\right) .
\end{aligned}
$$

The result follows.

In particular, taking $a=c=C=0, \gamma=p-1>0, A(x)>0$, yields the following corollary.

COROllary 3.1. Let $v>0$ and $u \geq 0$ be differentiable. Then

$$
L(u, v)=R(u, v) \geq 0,
$$

where

$$
\begin{aligned}
& L(u, v)=A(x)\left[\left|\nabla_{L} u\right|^{p}+(p-1)\left|\frac{u}{v} \nabla_{L} v\right|^{p}-p\left|\frac{u}{v} \nabla_{L} v\right|^{p-2} \nabla_{L} u \cdot\left(\frac{u}{v} \nabla_{L} v\right)\right], \\
& R(u, v)=A(x)\left|\nabla_{L} u\right|^{p}-\nabla_{L}\left(\frac{u^{p}}{v^{p-1}}\right) \cdot A(x)\left|\frac{u}{v} \nabla_{L} v\right|^{p-2} \nabla_{L} v .
\end{aligned}
$$

LEMMA 3.2. Suppose that $0<v \in C^{\infty}(\Omega)$ satisfies

$$
-L_{p, \alpha} v \geq \lambda g v^{p-1}
$$

for some $\lambda>0$ and weight function $g$ on $\Omega$, where

$$
-L_{p, \alpha} v=-\nabla_{L} \cdot\left(A(x)\left|\nabla_{L} v\right|^{p-2} \nabla_{L} v\right)
$$


Then, for any $u \in C_{0}^{\infty}(\Omega)$, one has

$$
\int_{\Omega} A(x)\left|\nabla_{L} u\right|^{p} \geq \lambda \int_{\Omega} g|u|^{p} .
$$

Proof. Let $\Omega_{0}$ be a compact subset of $\Omega$ and let $\phi \geq 0$ be differentiable. We have, by Corollary 3.1 ,

$$
\begin{aligned}
0 & \leq \int_{\Omega_{0}} L(\phi, v) \leq \int_{\Omega} L(\phi, v)=\int_{\Omega} R(\phi, v) \\
& =\int_{\Omega} A(x)\left|\nabla_{L} \phi\right|^{p}-\nabla_{L}\left(\frac{\phi^{p}}{v^{p-1}}\right) \cdot A(x)\left|\nabla_{L} v\right|^{p-2} \nabla_{L} v \\
& =\int_{\Omega} A(x)\left|\nabla_{L} \phi\right|^{p}+\frac{\phi^{p}}{v^{p-1}} \nabla_{L} \cdot\left(A(x)\left|\nabla_{L} v\right|^{p-2} \nabla_{L} v\right) \\
& \leq \int_{\Omega} A(x)\left|\nabla_{L} \phi\right|^{p}-\frac{\phi^{p}}{v^{p-1}} \lambda g v^{p-1} \\
& =\int_{\Omega} A(x)\left|\nabla_{L} \phi\right|^{p}-\lambda g \phi^{p} .
\end{aligned}
$$

For general $u$, let $\phi=u^{+}-u^{-}$. The proof is easily completed.

THEOREM 3.1. Let $A(z, t)=\frac{p-Q}{\beta p}\left|\frac{p-Q}{\beta p}\right|^{p-2} d^{(p-1)\left(1-\beta-\frac{Q}{p}\right)}$, with $\beta<0,1<p<Q$. Then for any $u \in C_{0}^{\infty}\left(\mathbb{R}^{n+m} \backslash(0,0)\right)$, we have

$$
\int_{\mathbb{R}^{n+m} \backslash(0,0)} A(z, t)\left|\nabla_{L} \phi\right|^{p} \geq\left(\frac{Q-p}{p}\right)^{p} \int_{\mathbb{R}^{n+m} \backslash(0,0)} \frac{|z|^{p \alpha}}{d^{k}}|u|^{p},
$$

where $k=Q-\frac{Q}{p}+p(\alpha+\beta)+(1-\beta)$.

Proof. Choosing $v=d^{\beta}$ and noting that

$$
\nabla_{L} v=\left(Z_{1} d^{\beta}, \ldots, Z_{n} d^{\beta}, Z_{n+1} d^{\beta}, \ldots, Z_{n+m} d^{\beta}\right)=\beta d^{\beta-1} \nabla_{L} d
$$

it follows that

$$
\left|\nabla_{L} v\right|=|\beta| d^{\beta-1}\left|\nabla_{L} d\right|=-\beta d^{\beta-1}\left|\nabla_{L} d\right|
$$

and

$$
\begin{aligned}
-\nabla_{L} & \left(A(z, t)\left|\nabla_{L} v\right|^{p-2} \nabla_{L} v\right) \\
= & -\nabla_{L} \cdot\left(\frac{p-Q}{\beta p}\left|\frac{p-Q}{\beta p}\right|^{p-2} d^{(p-1)\left(1-\beta-\frac{Q}{p}\right)}|\beta|^{p-2} d^{(\beta-1)(p-1)}\left|\nabla_{L} d\right|^{p-2} \beta \nabla_{L} d\right) \\
= & -\nabla_{L} \cdot\left(\frac{p-Q}{p}\left|\frac{p-Q}{p}\right|^{p-2} d^{-\frac{Q}{p}(p-1)}\left|\nabla_{L} d\right|^{p-2} \nabla_{L} d\right) \\
= & \left(\frac{Q-p}{p}\right)^{p-1} \nabla_{L} \cdot\left(|z|^{\alpha(p-2)} d^{-\frac{Q}{p}(p-1)-\alpha(p-2)} \nabla_{L} d\right)
\end{aligned}
$$




$$
\begin{aligned}
= & \left(\frac{Q-p}{p}\right)^{p-1}\left\{\alpha(p-2)|z|^{\alpha(p-2)-1} d^{-\frac{Q}{p}(p-1)-\alpha(p-2)} \cdot \nabla|z| \cdot \nabla_{L} d\right. \\
& +\left[-\frac{Q}{p}(p-1)-\alpha(p-2)\right] d^{-\frac{Q}{p}(p-1)-\alpha(p-2)-1}|z|^{\alpha(p-2)}\left|\nabla_{L} d\right|^{2} \\
& \left.+|z|^{\alpha(p-2)} d^{-\frac{Q}{p}(p-1)-\alpha(p-2)} L_{\alpha} d\right\},
\end{aligned}
$$

where we have used (2.2). Otherwise, using the formulae

$$
\begin{aligned}
\nabla_{L}|z| \cdot \nabla_{L} d= & \left(Z_{1}|z|, \ldots, Z_{n}|z|, Z_{n+1}|z|, \ldots, Z_{n+m}|z|\right) \\
& \cdot\left(d^{-2 \alpha-1}|z|^{2 \alpha} z_{1}, \ldots, d^{-2 \alpha-1}|z|^{2 \alpha} z_{n}, d^{-2 \alpha-1}|z|^{\alpha}(\alpha+1) t_{1},\right. \\
& \left.\ldots, d^{-2 \alpha-1}|z|^{\alpha}(\alpha+1) t_{m}\right) \\
= & \frac{d^{-2 \alpha-1}}{|z|}|z|^{2 \alpha}|z|^{2} \\
= & \frac{|z|^{2 \alpha+1}}{d^{2 \alpha+1}}
\end{aligned}
$$

$L_{\alpha} d=\frac{(Q-1)|z|^{2 \alpha}}{d^{2 \alpha+1}}$ and (2.2), (3.9) becomes

$$
\begin{aligned}
-\nabla_{L}( & \left.A(z, t)\left|\nabla_{L} v\right|^{p-2} \nabla_{L} v\right) \\
= & \left(\frac{Q-p}{p}\right)^{p-1}\left\{\alpha(p-2)|z|^{\alpha(p-2)-1} \frac{|z|^{2 \alpha+1}}{d^{2 \alpha+1}} d^{-\frac{Q}{p}(p-1)-\alpha(p-2)}\right. \\
& +\left[-\frac{Q}{p}(p-1)-\alpha(p-2)\right] d^{-\frac{Q}{p}(p-1)-\alpha(p-2)-1} \frac{|z|^{2 \alpha}}{d^{2 \alpha}}|z|^{\alpha(p-2)} \\
& \left.+|z|^{\alpha(p-2)} d^{-\frac{Q}{p}(p-1)-\alpha(p-2)} \frac{(Q-1)|z|^{2 \alpha}}{d^{2 \alpha+1}}\right\} \\
= & \left(\frac{Q-p}{p}\right)^{p-1}\left\{|z|^{p \alpha} d^{-Q+\frac{Q}{p}-\alpha p-1}\left[\alpha(p-2)+\frac{Q}{p}-Q-\alpha p+2 \alpha+Q-1\right]\right\} \\
= & \left(\frac{Q-p}{p}\right)^{p-1}\left\{|z|^{p \alpha} d^{-Q+\frac{Q}{p}-\alpha p-1-\beta(p-1)} v^{p-1}\left(\frac{Q}{p}-1\right)\right\} \\
= & \left(\frac{Q-p}{p}\right)^{p}|z|^{p \alpha} d^{-\left[Q-\frac{Q}{p}+p(\alpha+\beta)+1-\beta\right]} v^{p-1} .
\end{aligned}
$$

Hence we obtain the claim by Lemma 3.2.

REMARK. If $\beta=1-\frac{Q}{p}$, then (3.8) is (1.4) in [8].

4. A sharp Hardy-type inequality for $L_{\alpha}$. We first give a generalization of Theorem 8.3.4 in [2].

LEMMA 4.1. If there exists a function $0<f \in C^{\infty}$ such that $V(x) \geq \frac{L_{\alpha} f}{f}$ a.e. on $\mathbb{R}^{n} \times \mathbb{R}^{m}$, then $\operatorname{Spec}(H)$ (the spectrum of $\left.H\right) \subseteq[0, \infty)$, where $H f(x)=$ $-L_{\alpha} f(x)+V(x) f(x)$. 
Proof. Let $W=\frac{L_{\alpha} f}{f}, \varphi \in C_{0}^{\infty}$ and $\psi=\frac{\phi}{f}$. Then

$$
\begin{aligned}
Q(\phi) & =\int_{\mathbb{R}^{n+m}}\left(\left|\nabla_{L} \phi\right|^{2}+V|\phi|^{2}\right) d x \\
& \geq \int_{\mathbb{R}^{n+m}}\left(\left|\nabla_{L} \phi\right|^{2}+\frac{L_{\alpha} f}{f}|\phi|^{2}\right) d x \\
& \geq \int_{\mathbb{R}^{n+m}}\left(-\psi L_{\alpha} f-2 \nabla_{L} f \nabla_{L} \psi-f L_{\alpha} f+W f \psi\right) f \bar{\psi} d x \\
& =\int_{\mathbb{R}^{n+m}}\left(-\psi L_{\alpha} f-2 \nabla_{L} f \nabla_{L} \psi-f L_{\alpha} f+\frac{L_{\alpha} f}{f} f \psi\right) f \bar{\psi} d x \\
& =\int_{\mathbb{R}^{n+m}}\left(-2 f \bar{\psi} \nabla_{L} f \nabla_{L} \psi-f^{2} L_{\alpha} \psi \bar{\psi}\right) d x \\
& =\int_{\mathbb{R}^{n+m}}\left(-\bar{\psi} \nabla_{L} f^{2} \nabla_{L} \psi-\left(L_{\alpha} \psi\right)\left(f^{2} \bar{\psi}\right)\right) d x \\
& =\int_{\mathbb{R}^{n+m}}\left(-\bar{\psi} \nabla_{L} f^{2} \nabla_{L} \psi+\nabla_{L} \psi \nabla_{L}\left(f^{2} \psi\right)\right) d x \\
& =\int_{\mathbb{R}^{n+m}} \nabla_{L} \psi\left(-\bar{\psi} \nabla_{L} f^{2}+\nabla_{L}\left(f^{2} \bar{\psi}\right)\right) d x \\
& =\int_{\mathbb{R}^{n+m}}\left(\nabla_{L} \psi f^{2} \nabla_{L} \bar{\psi}\right) d x \\
& =\int_{\mathbb{R}^{n+m}} f^{2}\left|\nabla_{L} \psi\right|^{2} d x .
\end{aligned}
$$

Since $C_{0}^{\infty}$ is a form core, the conclusion is proved.

Theorem 4.1. Suppose that $u \in C_{0}^{\infty}\left(\mathbb{R}^{n} \times \mathbb{R}^{m}\right)$. Then

$$
\left(\frac{Q-2}{2}\right)^{2} \int_{\mathbb{R}^{n+m}} \frac{|z|^{2 \alpha}}{d^{2(\alpha+1)}} u^{2}(z, t) d z d t \leq \int_{\mathbb{R}^{n+m}}\left|\nabla_{L} u(z, t)\right|^{2} d z d t .
$$

Proof. Note

$$
L_{\alpha} f=\Delta_{z} f+|z|^{2 \alpha} \Delta_{t} f=\sum_{j=1}^{n} \frac{\partial^{2} f}{\partial z_{j}^{2}}+|z|^{2 \alpha} \sum_{j=1}^{m} \frac{\partial^{2} f}{\partial t_{j}^{2}} .
$$

For $s>0$ and $k<0$, choosing

$$
f=\left[s+|z|^{2(\alpha+1)}+(\alpha+1)^{2}|t|^{2}\right]^{k} .
$$

A direct calculation implies

$$
\begin{aligned}
\frac{\partial f}{\partial z_{j}}= & 2 k(\alpha+1)\left[s+|z|^{2(\alpha+1)}+(\alpha+1)^{2}|t|^{2}\right]^{k-1}|z|^{2 \alpha} z_{j}, \\
\frac{\partial^{2} f}{\partial z_{j}^{2}}= & 2 k(\alpha+1)\left[s+|z|^{2(\alpha+1)}+(\alpha+1)^{2}|t|^{2}\right]^{k-1}|z|^{2 \alpha} \\
& \times\left\{2(k-1)(\alpha+1)\left[s+|z|^{2(\alpha+1)}+(\alpha+1)^{2}|t|^{2}\right]^{-1}|z|^{2 \alpha} z_{j}^{2}+2 \alpha|z|^{-2} z_{j}^{2}+1\right\},
\end{aligned}
$$




$$
\begin{aligned}
\frac{\partial f}{\partial t_{j}}= & 2 k(\alpha+1)^{2} t_{j}\left[s+|z|^{2(\alpha+1)}+(\alpha+1)^{2}|t|^{2}\right]^{k-1}, \\
\frac{\partial^{2} f}{\partial t_{j}^{2}}= & 2 k(\alpha+1)^{2}\left[s+|z|^{2(\alpha+1)}+(\alpha+1)^{2}|t|^{2}\right]^{k-1} \\
& \times\left\{2(k-1)\left[s+|z|^{2(\alpha+1)}+(\alpha+1)^{2}|t|^{2}\right]^{-1}(\alpha+1)^{2} t_{j}^{2}+1\right\} .
\end{aligned}
$$

Combining (4.4) and (4.6) with (4.2) leads to

$$
\begin{aligned}
L_{\alpha} f= & 2 k(\alpha+1)\left[s+|z|^{2(\alpha+1)}+(\alpha+1)^{2}|t|^{2}\right]^{k-1}|z|^{2 \alpha} \\
& \times\left\{\sum_{j=1}^{n} 2(k-1)(\alpha+1)\left[s+|z|^{2(\alpha+1)}+(\alpha+1)^{2}|t|^{2}\right]^{-1}|z|^{2 \alpha} z_{j}^{2}\right. \\
& +\sum_{j=1}^{m} 2(k-1)(\alpha+1)\left[s+|z|^{2(\alpha+1)}+(\alpha+1)^{2}|t|^{2}\right]^{-1}(\alpha+1)^{2} t_{j}^{2} \\
& \left.+2 \alpha|z|^{-2} z_{j}^{2}+1+(\alpha+1)\right\} \\
= & 4 k(k-1)(\alpha+1)^{2}\left[s+|z|^{2(\alpha+1)}+(\alpha+1)^{2}|t|^{2}\right]^{k-2}|z|^{2 \alpha}\left[|z|^{2(\alpha+1)}+(\alpha+1)^{2}|t|^{2}\right] \\
& +2 k(\alpha+1)\left[s+|z|^{2(\alpha+1)}+(\alpha+1)^{2}|t|^{2}\right]^{k-1}|z|^{2 \alpha}[m(\alpha+1)+n+2 \alpha], \\
\frac{L_{\alpha} f}{f}= & \frac{\left[4 k^{2}(\alpha+1)^{2}+2 k(\alpha+1)(m(\alpha+1)+n-2)\right]}{\left[s+|z|^{2(\alpha+1)}+(\alpha+1)^{2}|t|^{2}\right]^{2}} \\
& \times|z|^{2 \alpha}\left[|z|^{2(\alpha+1)}+(\alpha+1)^{2}|t|^{2}\right] \\
& +\frac{2 k(\alpha+1)|z|^{2 \alpha}[m(\alpha+1)+n+2 \alpha] s}{\left[s+|z|^{2(\alpha+1)}+(\alpha+1)^{2}|t|^{2}\right]^{2}} .
\end{aligned}
$$

Denote

$$
\begin{aligned}
g(k) & =4(\alpha+1)^{2} k^{2}+2(\alpha+1)[m(\alpha+1)+n-2] k \\
& =4(\alpha+1)^{2} k^{2}+2(\alpha+1)(Q-2) k .
\end{aligned}
$$

Clearly, taking $k=-\frac{Q-2}{4(\alpha+1)}$, one has

$$
\begin{aligned}
\min g(k) & =4(\alpha+1)^{2}\left[-\frac{Q-2}{4(\alpha+1)}\right]^{2}+2(\alpha+1)(Q-2)\left[-\frac{Q-2}{4(\alpha+1)}\right] \\
& =\frac{(Q-2)^{2}}{4}-\frac{(Q-2)^{2}}{2} \\
& =-\left(\frac{Q-2}{2}\right)^{2}
\end{aligned}
$$


and

$$
\begin{aligned}
\frac{L_{\alpha} f}{f}= & -\left(\frac{Q-2}{2}\right)^{2} \frac{\left[|z|^{2(\alpha+1)}+(\alpha+1)^{2}|t|^{2}\right]|z|^{2 \alpha}}{\left[s+|z|^{2(\alpha+1)}+(\alpha+1)^{2}|t|^{2}\right]^{2}} \\
& +\frac{2 k(\alpha+1)|z|^{2 \alpha}[m(\alpha+1)+n+2 \alpha] s}{\left[s+|z|^{2(\alpha+1)}+(\alpha+1)^{2}|t|^{2}\right]^{2}} \leq 0 .
\end{aligned}
$$

By Lemma 4.1, we have

$$
\begin{aligned}
\int_{\mathbb{R}^{n+m}} & {\left[\left(\frac{Q-2}{2}\right)^{2} \frac{\left[|z|^{2(\alpha+1)}+(\alpha+1)^{2}|t|^{2}\right]|z|^{2 \alpha}}{\left[s+|z|^{2(\alpha+1)}+(\alpha+1)^{2}|t|^{2}\right]^{2}}\right.} \\
& \left.-\frac{2 k(\alpha+1) s|z|^{2 \alpha}[m(\alpha+1)+n+2 \alpha]}{\left[s+|z|^{2(\alpha+1)}+(\alpha+1)^{2}|t|^{2}\right]^{2}}\right] u^{2}(z, t) d z d t \\
\leq & \int_{\mathbb{R}^{n+m}}\left|\nabla_{L} u(z, t)\right|^{2} d z d t
\end{aligned}
$$

Letting $s \rightarrow 0$, we deduce the claim.

\section{REFERENCES}

1. M. S. Baouendi, Sur une classe d'opérateurs elliptiques dégénérés, Bull. Soc. Math. France 95 (1967), 45-87.

2. E. B. Davies, Spectral theory and differential operators (Cambridge University Press, 1996).

3. J. A. Goldstein and Q. S. Zhang, On a degenerate heat equation with a singular potential, J. Functional Analysis 186 (2001), 342-359.

4. N. Garofalo, Unique continuation for a class of elliptic operators which degenerate on a manifold of arbitrary codimension, J. Differential Equations 104 (1993), 117-146.

5. N. Garofalo and D. M. Nhieu, Isoperimetric and Sobolev inequalities for CarnotCaratheodory spaces and the existence of minimum surfaces, Comm. Pure Appl. Math. 49 (1996), 1081-1144. $458-476$.

6. V. V. Grushin, On a class of hypoelliptic operators, Math. Sbornik, USSR 12 (1970),

7. T. Kusauo, J. Jaros and N. Yoshida, A Picone-type identity and Sturmian comparison and oscillation theorems for a class of half-linear partial differential equations of second order, Nonlinear Anal. 40 (2000), 381-395.

8. H. Zhang and P. Niu, Picone identity and Hardy inequality for a class of vector fields, J. Math. (Wuhan) 23 (2003), 121-125 (Chinese). 